\section{Bioactive Follicle-Stimulating Hormone}

\author{
Inese Z. Beitins and Vasantha Padmanabhan
}

Specific and sensitive in vitro bioassays suitable for measurement of follicle-stimulating hormone (FSH) in small quantities of serum and urine have recently been validated. Comparisons of bioassay (B-FSH) potencies with immunoassay (I-FSH) estimates and chromatofocusing patterns have facilitated new discoveries regarding the regulation of FSH biosynthesis, storage, secretion, serum concentrations and target cell responsiveness, plasma clearance, and the significance of excreted forms. A review of recent studies reveals that luteinizing hormone and FSH, as well as B-FSH and I-FSH, appear to be differentially regulated by GnRH, GnRH agonists and antagonists, sex steroids and gonadal peptides in both sexes, with significant differences between the sexes. The results support the overall hypotheses that the quality as well as the quantity of FSH are significant contributors to target cell signal transduction and that distinct FSH isoforms with different functional capabilities influence the expression of acule (ovulation) and chronic reproductive events. (Trends Endocrinol Metab 1991; 2:145-151)

Research during the last two decades has firmly established that follicle-stimulating hormone (FSH), like other glycoproteins, is not a unique protein, but rather a family of heterogeneous isoforms of varying immunologic and bioactive potencies. Such mixes of isoforms are found within the pituitary, resulting from differential promoter action, alternative splicing, induction of point mutations during biosynthesis, or posttranslational changes, including carbohydrate incorporation prior to or during granule formation within the gonadotrope. Upon secretion, these molecular variations determine tertiary structure and metabolic clearance. Molecules lacking sialic acid residues (relatively less acidic) have shorter half-lives within the circulation,

Inese Z. Beitins and Vasantha Padmanabhan are at the Division of Pediatric Endocrinology at the University of Michigan, Ann Arbor, MI 48109-0718, USA. by remaining toxins or foreign proteins within the test materials, thus leading to poor reproducibility of results. Though the assays incorporated metabolic clearance, they were relatively insensitive compared with immunoassays. With immunoassay development, standards and antibodies were preferentially selected that would provide the desired sensitivity and exhibit parallel responses to the test materials. It became evident early, however, that comparisons of in vivo bioassay responses of extracted rat pituitary FSH to immunoassay potencies did not provide consistent bioactive to immunoreactive (B-I) ratios. Furthermore, steroid treatment of castrated rats altered the B-I ratio of FSH as well as its clearance (Bogdanove et al. 1973). These studies led to the hypothesis that both GnRH stimulation and steroid modulation altered not only the quantity but also the quality of the hormone (Chappel et al. 1983).

These initial observations were extended to other animal species, such as the hamster (Chappel et al. 1983). A series of experiments studying FSH isoform distribution patterns following chromatofocusing and measuring hormonal potencies by means of receptor binding, in vitro bioassay and immunoassay methods confirmed that GnRH stimulation and sex steroid modulation, as in the estrous cycle or throughout puberty, induced shifts within the FSH isoform distribution patterns. Shifts from the acidic to relatively more basic isoforms also resulted from neuraminidase treatment, confirming the importance of the presence of sialic acid upon the $\mathrm{pI}$ of the FSH molecule.

Though sensitive and precise in vitro bioassays utilizing rat interstitial cell testosterone production (RICT) had been validated and widely used for measurements of dynamic changes in the B-I ratios of luteinizing hormone (LH) in a variety of physiologic and pathophysiologic conditions (Dufau and Veldhuis 1987), the development of similar in vitro bioassays suitable for measurement of FSH in unextracted serum lagged behind. In rat granulosa cells, aromatase could be reliably induced by FSH standards, yet the addition of serum to the in vitro system depressed the biologic response and led to nonparallelism. This problem was overcome by treating serum with $12 \%$ polyethylene glycol (PEG) (Jia and Hsueh 
1985). We, on the other hand, optimized the conditions of the rat Sertoli cell aromatase induction assay first described by Van Damme et al. (1979). By improving the sensitivity severalfold, we are now able to reliably determine FSH in unextracted serum (Padmanabhan et al. 1987) and urine.

Building upon the experience with bioassayed (B)-LH and its comparisons with immunologic potencies, which addressed sex differences, regulation of secretion by $\mathrm{GnRH}$ and modulation by sex steroids, plasma clearance, and responses to GnRH agonists and antagonists, we now describe similar experiences with the newly validated in vitro FSH bioassays. Because these in vitro bioassays, unlike the immunoassays, have only limited species specificity, they can be utilized in animal models either to confirm regulatory mechanisms impossible to study in the human or chart new territory in reproductive endocrinology in a variety of species where FSH measurements were previously not possible.

Within our own work, we have tested the overall hypothesis, which was first proposed by Chappel et al. (1983), that FSH isoforms have different physiologic functions, with the sialylated (longer half-lived, but less reactive in the in vitro assay) playing an important role in organ growth and the maintenance of reproductive function, whereas the relatively more basic (less sialylated, short half-lived) play an important role in dynamic events such as ovulation.

\section{- Comparison of Bioassays of FSH and B-FSH Serum Concentrations in Physiologic States}

The major differences between the granulosa aromatase induction assay (GABA) and Sertoli cell aromatase assay, which have been recently reviewed (Wang 1988; Hsueh et al. 1989), are (a) the selection of target tissues from rats of different sexes and reproductive ages; (b) pretreatment of serum, but not urine, with PEG in the GABA, but not Sertoli cell assay; (c) the choice of standards with LER 907, IRP 78/549, Pergonal, and NIH-hFSH-3 used by various investigators for GABA, but the pituitary standards (NIH-hFSH-2 and NIH-hFSH-3) for the Sertoli cell assay; and (d) the expression of B-FSH potencies as $\pi / L$ as related to the in vivo FSH biopotency of the World Health Organi- zation Reference Preparation for the GABA and micrograms per liter of purified standard for the Sertoli cell assay. Without actual comparisons of B-FSH potencies of the same preparations in both assay systems, it is not valid to convert the values obtained in one to those obtained with a different standard in the other. Similar discrepancies in the selection of antibodies, standards, and assay conditions exist within the respective FSH immunoassays. Therefore, though the results from various studies cannot be compared quantitatively, trends in circulating and excreted levels of B-FSH can be established and comparcd. Likcwisc, variations in the FSH B-I ratio within each assay system can reflect variable FSH isoform distribution, which can be confirmed by chromatofocusing. Physiologic validation of both assays (GABA and Sertoli cell) was accomplished by measurement of BFSH in single serum samples obtained

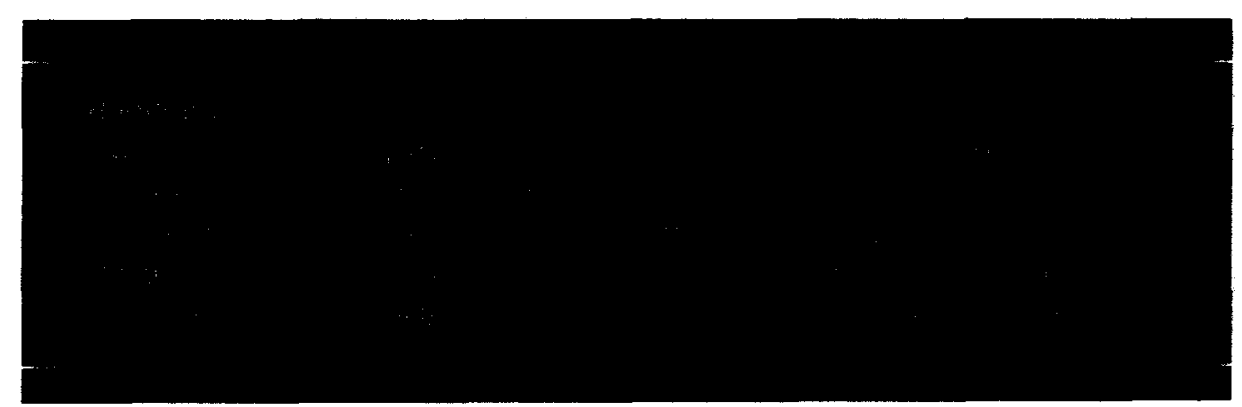

from cross-sectional studies in men and women. B-FSH was not detected in the sera from hypophysectomized patients or women treated with oral contraceptives. With both assays, serum B-FSH values were lowest in men, followed by women during the luteal and follicular phases of their menstrual cycles. Sera from postmenopausal women and women with premature ovarian failure contained the highest B-FSH concentrations, which exceeded values observed during the midcycle of menstruating women.

\section{- Serum B-FSH Levels and FSH B-I Ratios in Men}

With the GAB assay, cross-sectional studies have been performed comparing serum B-FSH concentrations and B-I ratios in normal young men, elderly men, and men with idiopathic azoospermia, oligospermia, and prostatic cancer. The effects of GnRH agonist and antago- nist therapy as well as orchidectomy have also been reported. Young men have circulating B-FSH concentrations of $7.7 \pm 2.0 \mathrm{IU} / \mathrm{L}$ (mean $\pm \mathrm{SE}$ ), with a mean B-I ratio of $2.7 \pm 0.3$ (Tenover et al. 1987). Elderly men and those with idiopathic azoospermia have identical mean serum B-FSH concentrations, but, owing to increased serum I-FSH levels, lower mean serum FSH B-I ratios (1.4 \pm 0.2 and $1.5 \pm 0.5$, respectively) (Tenover et al. 1987; Wang et al. 1987). Men with severe oligospermia, on the other hand, had elevated serum B- and I-FSH concentrations, with a B-I ratio of $1.7 \pm 0.3$. Elderly men with prostatic carcinoma had mean scrum B-FSH concentrations of $19.7 \pm 4.1 \mathrm{IU} / \mathrm{L}$, with a B-I ratio of $1.7 \pm 0.2$ (Huhtaniemi et al. 1988). Treatment with a GnRH analogue decreased serum I-FSH concentrations to a greater extent than serum B-FSH concentrations, with a resulting $\mathrm{B}-\mathrm{I}$ ratio of $6.7 \pm 0.8$ in $2-3$ weeks of treatment

and a gradual decline to pretreatment B-I ratio levels by 6 months. Orchidectomy at this point caused an initial increase in 2 weeks to a mean serum FSH B-I ratio of 6.6-6.8, with a decline toward pretreatment ratios during the next 3 months. In normal men treated with $\mathrm{GnRH}$ agonists, with or without testosterone enanthate, the serum FSH B-I ratios remained unchanged (Pavlou et al. 1988). Five men treated with a potent GnRH antagonist clearly demonstrated that serum B-FSH levels were suppressed to a greater extent than were serum I-FSH levels (Dahl et al. 1986). These effects led to a dramatic lowering of the serum FSH B-I ratio. Several conclusions could be reached: (a) serum $B$ and I-FSH concentrations did not increase or decrease in parallel in various pathophysiologic conditions; (b) in older men, or those with inadequate spermatogenesis, the serum FSH B-I ratio was significantly lower than in 
younger men or following orchidectomy; (c) treatment with GnRH agonists failed to decrease serum B-FSH concentrations to the same degree as serum I-FSH or I-LH concentrations; and (d) treatment with $\mathrm{GnRH}$ antagonists preferentially depressed serum B-FSH over I-FSH. It therefore appeared that serum B-FSH synthesis and secretion were differentially regulated from that of $\mathrm{LH}$ and that testicular factors depressed B-FSH secretion. These inhibitory effects were removed upon orchidectomy.

\section{- Serum B-FSH Concentrations and FSH B-I Ratios in Women}

In postmenopausal women and women with premature ovarian failure, both $\mathrm{B}$ and I-FSH concentrations were elevated, with the resulting serum FSH B-I ratios not significantly different from those of cycling women in the follicular phase or young men (Wang 1988). Treatment with varying doses of a potent $\mathrm{GnRH}$ antagonist resulted in a $51 \%-63 \%$ decrease in serum I-LH and a $14 \%-33 \%$ decrease in I-FSH. B-LH and B-FSH were suppressed to a greater extent than the corresponding immunoreactive gonadotropins (Mortola et al. 1989). This resulted in a $27 \%$ decline in the B-I ratio for FSH

Serum B-FSH concentrations have been determined in daily blood samples obtained throughout the menstrual cycle by utilizing the GABA (Jia et al. 1986; Fauser et al. 1989) and the Sertoli cell assay (Padmanabhan et al. 1988b; Beitins et al. 1990). With the GABA, the serum B-FSH concentrations paralleled those of serum I-FSH, with no change in the FSH B-I ratio throughout all phases of the menstrual cycle. The FSH B-I ratio ranged from 1.4 to 3.4 , with a mean of 2.5. Urinary B-FSH levels, obtained in daily first morning void samples, however, exhibited marked elevations in urinary B-FSH concentrations, which coincided with the LH peak (Dahl et al. 1987). There was a highly significant correlation between serum and urinary B-FSH. Infusion of an antagonist of $\mathrm{GnRH}$ at $30 \mu \mathrm{g} / \mathrm{kg} \cdot \mathrm{h}$ in women during the midfollicular phase suppressed $B$ FSH levels by $64 \%$, but I-FSH only by $29 \%$. When the GnRH antagonist was given subcutaneously at a higher dose of $80 \mu \mathrm{g} / \mathrm{kg}$ twice daily for 3 days during the midfollicular phase, the B-FSH response was biphasic, with a maximal decrease on the second day, returning to basal levels by the third (Kessel et al. 1988).

Having used the Sertoli cell assay, Padmanabhan et al. (1988b) reported that women during the menstrual cycle had significantly higher B-FSH concentrations during the midcycle than the follicular phase. The serum FSH B-I ratio was the highest during this phase of the menstrual cycle and the lowest during the luteal phase. These changes in serum B-FSH reflected shifts in the distribution of FSH isoforms from the acidic to a relatively basic pI (with higher B-I ratios) upon chromatofocusing. These results were compatible with the hypothesis that less sialylated, shorter-lived FSH isoforms circulated during the midcycle period and may participate in dynamic functions such as ovulation. The relative shift of the FSH isoform biosynthesis and secretion could be due to either increased GnRH pulse frequency or the rising sex steroid values. These potent FSH isoforms could participate in the induction of plasminogen activator, activating plasmin, finally leading to the breakdown of collagen fibers present in the tissues of the ovulating follicles (Hsueh et al. 1989).

During the menstrual cycle studies, several women had become pregnant. Serum B-FSH levels, in contrast to IFSH levels, increased significantly from postovulation day 7 to the end of the menstrual cycle study period. The serum FSH B-I ratio increased from 2.5 on the LH peak day to 70 by day 15 after the $\mathrm{LH}$ peak day. In another study, blood samples had been obtained every $10 \mathrm{~min}$ for $10 \mathrm{~h}$ on days 10,20 , and 27 after the LII peak in a pregnant woman. The serum B-FSH concentrations appeared pulsatile and the FSH B-I ratio was likewise elevated. This FSH-like bioactive material was not due to cross reaction with hCG, $\beta$ subunit, $\alpha$-hCG, $\alpha$-fetoprotein, or human placental lactogen. Removal of steroids by charcoal extraction did not alter the final results. FSH-like activity was present in the media from placental incubates, with an FSH B-I ratio of 47.1 (Padmanabhan et al. 1989a). FSH-like bioactive material was also secreted by choriocarcinoma cells in culture. The increase of B-FSH in pregnancy serum was confirmed by FSH isoform distribution patterns. There was a progressive increase in serum B-FSH-like activity until term, but 6 weeks postpartum B-FSH levels were low and did not increase with $\mathrm{GnRH}$ stimulation.

\section{- Serum B-FSH Concentrations During Development and Puberty}

In human fetuses, who have had chordocentesis for medical indications but were found to be normal, serum B-FSH concentrations were higher in girls than boys at 20-25 weeks of gestation, but declined and were no longer significantly different between the sexes at 35 and 40 weeks of gestation (unpublished data). In samples drawn simultaneously from the mother and by chordocentesis, the maternal B-FSH levels were - 3 times higher than in the fetus. The B-FSH serum concentrations during fetal life near term were -10 times higher than those during pubertal development in boys and girls.

From cross-sectional as well as longitudinal studies of bioactive gonadotropin concentrations throughout puberty, though B-LH levels rose progressively through each stage of puberty, B-FSH serum concentrations did not change significantly (Reiter 1982; Beitins et al. 1990). The serum B-FSH concentrations were as high during the prepubertal period as during later stages of puberty when both boys and girls had matured and sex steroid concentrations were significantly increased. Serum I-FSH concentrations were significantly higher during puberty in girls than boys, with the resulting serum FSH B-I ratio significantly lower in girls than boys. Serum B-FSH levels during puberty were not significantly different from those of men and women during the follicular phase of the menstrual cycle, but were lower than during the periovulatory period. Similar findings have been reported in girls with precocious puberty (Wang et al. 1990). Basal serum B-FSH levels were not significantly different in patients with precocious puberty, premature thelarche, or prepubertal controls. After 12 months of GnRH agonist therapy, both I-FSH and B-FSH concentrations were suppressed in patients with precocity. The serum FSH B-I ratio was not changed. In 12 volunteers from ages 5-12 years, however, overnight urinary excretion of B-FSH, expressed per milligram of creatinine, revealed a gradual increase from childhood to pre- and 
postmenarchal period (Fauser et al. 1989). The number of subjects per group was small $(n=3)$ and the differences in urinary concentrations increased less than twofold.

\section{- Regulation of B-FSH Secretion}

\section{Role of $\mathrm{GnRH}$}

From the preceding studies, it is apparent that GnRH and GnRH agonists and antagonists influence B- and I-FSH secretion and potency. This was further confirmed by administering small pulses of GnRH $(0.025 \mu \mathrm{g} / \mathrm{kg})$ intravenously at hourly intervals for 3-5 days to boys with idiopathic hypogonadotropic hypogonadism (Padmanabhan et al. 1988a). Serum B-FSH concentrations increased significantly after $91 \%$ of the GnRH doses, even at times when there were no increases in serum I-FSH, I-LH, or sex steroid concentrations. The serum B-FSH responses occurred consistently within $20 \mathrm{~min}$ after the GnRH administration, but the B-FSH levels had returned to baseline by $60 \mathrm{~min}$, indicating rapid clearance of the newly secreted B-FSH.

In midpubertal boys who had blood withdrawn every $10 \mathrm{~min}$ from 2000 to $0800 \mathrm{~h}, \mathrm{~B}-\mathrm{FSH}$ was secreted in clearly discernible pulses before, during, and after the onset of sleep (Hassing et al. 1990). In association with the increased frequency and amplitude of I-LH pulses, there was a minimal, but significant, increase in mean B-FSH levels $(p=0.046)$, pulse frequency $(p=0.045)$, but not BFSH pulse amplitude. When testosterone was infused at the rate of the adult male production rate, the mean serum I-LH rise and nocturnal increase in $\mathrm{LH}$ pulse frequency were abolished without an effect on B-FSH pulsatility.

To dissect the relative contribution of GnRH versus steroids further, nutritionally growth-retarded ovariectomized lambs were studied during nutritional repletion (Padmanabhan et al. 1989b). In this animal model, LH secretory pulses were utilized as an index of endogenous GnRH secretion. During the period of nutritional restriction, I-LH and I-FSH levels were low and relatively apulsatile in serial blood samples obtained every 12 min for $4 \mathrm{~h}$. In contrast, serum B-FSH showed a pulsatile secretory pattern that became even more apparent with nutritional repletion. In contrast to increasing I-LH pulse frequency, FSH pulse frequency remained stable, whereas FSH pulse amplitude increased. Serum B-FSH pulse amplitudes were $57 \pm 7 \%$ greater than I-FSH pulses. The asynchronous occurrence of FSH pulses in the absence of I-LH pulses led us to speculate that FSH may be more sensitive to small changes in $\mathrm{GnRH}$ secretion than I-LH or that a hypothalamic releasing factor specific for FSH may be present.

\section{Effect of Steroids on B-FSH Secretion}

In addition to the study with the acute testosterone infusion in midpubertal boys described above, the effects of acute ( 24 h) infusions and long-term steady-state infusions of dihydrotestosterone (DHT) and estradiol $\left(\mathrm{E}_{2}\right)$ in normal men have been performed. Men infused with $E_{2}$ to elevate their mean serum $E_{2}$ to twice their endogenous $E_{2}$ levels for $24 \mathrm{~h}$ did not have a significant change in their mean serum B-FSH levels. Likewise, when DHT or $\mathrm{E}_{2}$ was infused for a total of $5^{1 / 2}$ days and blood withdrawn every $10 \mathrm{~min}$ for the last $28 \mathrm{~h}$, the mean serum B-FSH concentrations in pooled samples were not significantly different whether measured with the Sertoli cell aromatase induction or GABA (Urban et al. 1990). Therefore, it appeared that neither $T$, DHT, or $\mathrm{E}_{2}$ significantly altered mean B-FSH serum concentrations in midpubertal boys and men.

In women with gonadal dysgenesis treated with estrogenic steroids for 21 days, there was an initial parallel decline in the B-and I-FSH levels for about the first 14 days with a stable serum FSH B-I ratio. During week 3 , however, I-FSH levels continued to decline while serum B-FSH levels remained stable, leading to a significant increase in the serum FSH B-I ratio (Padmanabhan et al. 1988b). This increase in the FSH B-I ratio was similar to that observed during the periovulatory period of the menstrual cycle, when both GnRH pulse frequency and amplitude as well as serum $E_{2}$ levels were increasing.

In women with polycystic ovary syndrome (PCOS), endogenous serum androgens are elevated. Irrespective of whether the patients had elevated baseline and post-GnRH stimulation I-LH levels or whether they were relatively normal (group II), mean scrum B-FSH concentrations were higher than serum
I-FSH concentrations and similar to levels found in normal women during the follicular phase (unpublished data) When $E_{2}$ and progesterone were replaced at luteal concentrations for 20 days, mean serum I-FSH concentrations decreased significantly $(2.3 \pm 0.2$ to $0.6 \pm 0.1 \mu \mathrm{g} / \mathrm{L}$ ), while mean serum BFSH concentrations remained stable $(3.4 \pm 0.2$ vs $3.1 \pm 0.3 \mu \mathrm{g} / \mathrm{L})$. The resulting serum FSH B-I ratio increased from $1.6 \pm 0.1$ to $7.0 \pm 2.6 \quad(p<0.01)$ The mean serum B-FSH concentrations in the presence of luteal phase levels of $\mathrm{E}_{2}$ and progesterone were significantly higher than during the spontaneous luteal phases $(1.4 \pm 0.2 \mu \mathrm{g} / \mathrm{L})$. These results indicated that serum B-FSH levels in PCOS were slightly higher than in normal controls but did not suppress with luteal phase $E_{2}$ and $P$ administration in a manner similar to serum I-FSH. The low serum B-FSH levels during the spontaneous luteal phase were probably related to secretions from the corpus luteum itself.

\section{- FSH Isoform Distribution Patterns and FSH Clearance in the Human}

FSH isoform distribution patterns are difficult to obtain from unaltered human tissues and biologic fluids. Pituitaries obtained postmortem are subject to autolysis and those removed because of tumors may not synthesize normal hormonal isoforms. Plasma concentrations of FSH are relatively low. Nevertheless, FSH isoform distribution patterns from FSH-secreting tumors confirmed animal studies and revealed that the highest FSH B-I ratios were present in the less acidic fractions of the chromatofocused eluates (Padmanabhan et al. 1988b). FSH extracted from pituitaries was qualitatively different in young women from FSH derived from men and elderly women (Wide and Hobson 1983). FSH isoform distribution patterns from chromatofocused serum revealed an increase in the relatively less acidic FSH isoforms during the periovulatory period as well as following chronic estrogenic therapy in women with gonadal dysgenesis. Therefore, during high estrogenic states, circulating FSH isoforms are less acidic (less sialic acid) and have a shorter circulation time, but retain binding to target tissue receptors. Conversely, during states of low estro- 
genic stimulation, such as the follicular phase of the menstrual cycle (Padmanabhan et al. 1988b), men, postmenopausal women, and castrated men (Wide and Hobson 1983), the charge of the FSH is relatively more acidic. Treatment with neuraminidase eliminated the FSH charge differences (Wide 1986). GnRH stimulation in patients with Turner's syndrome likewise induced the appearance of less negatively charged FSH isoforms in electrophoretic mobility studies (Wide and AlbertssonWiklund 1990). Finally, infusion of a GnRH antagonist into hypogonadal women increased basic FSH isoforms, as determined by chromatofocusing (Dahl et al. 1988a). These isoforms bound to granulosa cell FSH receptors, but were devoid of biologic activity and, in fact, inhibited FSH action.

In addition to the differential binding to FSH receptors and induction of biologic responsiveness, different FSH isoforms are cleared from the circulation at different rates. FSH extracted from pituitaries of young women was cleared from the circulation of mice at a faster rate than FSH from men or elderly women (Wide and Wide 1984). Studies combining studies of molecular mobility on electrophoresis with metabolic clearance rate determined that the less negatively charged FSH isoforms are cleared more rapidly (Wide 1986). Therefore, the charge ( $\mathrm{pI}$ or sialic acid content) regulates the survival of FSH within the circulation.

From the studies utilizing in vitro FSH bioassays, the disappearance of newly secreted FSH in response to GnRH in patients with idiopathic hypogonadotropichypogonadism is $\sim 60 \mathrm{~min}$ ( $\mathrm{Pad}$ manabhan et al. 1988a). This is in contrast to the determinations based on I-FSH measurements after hypophysectomy or bolus injection, where the plasma half-life was stated to be $240 \mathrm{~min}$. We have recently calculated the half-life of purified human FSH (Metrodin) during continuous steady-state infusions in hypogonadotropic men. The FSH half-life for this material was $3.2 \mathrm{~h}$ by in vitro bioassay for a one-component fit and 0.9 $\mathrm{h}$ (fast) and $8.3 \mathrm{~h}$ (slow) for a twocomponent fit (unpublished data). The half-life of endogenous and exogenous infused FSH appears to be more rapid when estimated by in vitro bioassay than by immunoassays.

\section{- Urinary B-FSH in Various Species}

In vitro FSH bioassays can be utilized in a variety of animal species. We have validated the assay for the measurement of B-FSH in sera of sheep (Padmanabhan et al. 1989b). With the GABA for unextracted small quantities of urine, urinary B-FSH levels have been measured in rats during puberty (Dahl et al. 1988b), lowland gorillas (Dahl and Hsueh 1987), giant panda (Montfort et al. 1989), and killer whales (Walker et al. 1988). In all of these animal species, urinary estimates integrated pulsatile serum patterns (if they existed) and reduced the stress of handling and venipuncture. Urinary B-FSH was measured in male and female rats from birth to 40 days of age. Detectable urinary B-FSH levels were present during the neonatal period and throughout puberty. The urinary B-FSH profiles were highly correlated with serum B-FSH $(r=0.93)$. Urinary concentrations, however, were 50 times higher than in serum. In the lowland gorilla, a midcycle B-FSH surge was detected. Two peaks of FSH were present during the follicular phase in the gorilla as well as the giant panda. The latter likewise had a urinary periovulatory FSH peak. A similar bimodal urinary B-FSH pattern was found in the killer whale. This method of measurement therefore provides useful information regarding reproductive hormones, their regulation, and their reproductive cyclicity in endangered species and marine mammals. This information can be skillfully utilized in the management and breeding programs of these animals.

The reasons for the greater urinary than serum B-FSH concentrations (100fold during the menstrual cycle in women and 50-fold during maturation in the rat) are not known (Dahl et al. 1988b). Customarily, in human studies, to satisfy the principle of using the standard derived from the same source as the test material, pituitary FSH standards have been used for serum FSH determinations and urinary FSH for urinary FSH estimates. Also, the methods of extraction for the Steelman-Pohley in vivo bioassay and immunoassay are different. Nevertheless, with all these reservations, we have found the urinary FSH B-I ratio to be $1.16 \pm 0.11$ in postmenopausal women (Beitins et al. 1980). We have not yet made similar comparisons using the in vitro bioassay for FSH. Much additional work regarding FSH clearance needs to be performed to determine whether the urine contains a large proportion of the desialylated rapidly cleared FSH isoforms that still retain in vitro bioactivity. Thus, during puberty, when the urinary B-FSH levels have been shown to increase, but the serum B-FSH concentrations remain stable, urinary B-FSH determinations may shed more light upon the types of FSH isoforms that circulate and are excreted than the arduous task of performing chromatofocusing studies in sera with low values of B-FSH.

\section{- Summary}

The availability of the in vitro FSH bioassay enabled, for the first time, the beginning of studies regarding the neuroendocrine regulation of FSH biosynthesis and secretion according to models widely utilized for LH (I-LH pulse characteristics and B-LH circulatory patterns). The ability to determine B-FSH pulses in serum has revealed that LH and FSH are differentially regulated and released in animals and man. In addition, studies with GnRH agonists and antagonists reveal differential regulation of $B$ and I-LH secretion. During pregnancy, likewise, high circulating levels of B-FSH-like activity with low immunoreactivity are present. Chromatofocusing patterns of serum have confirmed that changes in the FSH B-I ratio are associated with shifts in the FSH isoform patterns. Estrogenic stimulation (midcycle, therapy) increases serum FSH B-I ratio in women, but not men, and is associated with an increase in the rela tively less acidic FSH isoforms. Gonadal peptides such as inhibin in both sexes may modulate B-FSH secretion. Therefore, the overall regulation of gonadotropin synthesis, release, circulation time, receptor binding, and signal transduction is regulated at the pituitary gonadotrope. Small GnRH signals (in frequent and/or low amplitude) appear to promote FSH synthesis and release preferentially until the signal has achieved the magnitude required for $\mathrm{LH}$ secretion. Then, with further maturation, gonadal peptides modulate FSH secretion, resulting in relatively stable mean FSH values while LH secretion is augmented. Sex steroids, on the other hand, modulate LH secretion to a greater extent than FSH. 


\section{- Future Perspectives}

Exciting studies are under way by several investigators utilizing recombinant FSH from cotransfection of the common $\alpha$ and FSH $\beta$ genes into eukaryotic cell lines (Hsueh et al. 1989). The recombinant FSH is bioactive and has an almost identical $\mathrm{pI}$ as the purified pituitary FSH standard (NIADDK-hFSH-I-3). By utilizing this methodology, large quantities of purified bioactive FSH can be made to be used as standards in assays, as well as physiologic and future clinical studies. In addition, by making single aminoacid substitutions in crucial points within the FSH sequence, alterations in glycosylation and tertiary structure can be induced. These mutants provide unparalleled opportunities for structure-function studies. Once the true importance and role of the various FSH isoforms is determined, it will be possible to design FSH isoforms for desired functions: sialylated, long-lived for growth and maintenance of reproductive organs; desialylated, short-lived for acute dynamic events, such as ovulation; and antagonistic FSH isoforms for potential use in contraception.

\section{- Acknowledgments}

This work was supported by grants HD-18515 and HD-23812. The authors thank Dr. M. Dufau for her encouragement and support and Mrs. Jeanne Townsend for her excellent typing skills.

References

Beitins IZ, Shah A, O'Loughlin K, et al.: 1980. The effects of fasting on serum and urinary gonadotropins in obese, postmenopausal women. J Clin Endocrinol Metab 51:26-34.

Beitins IZ, Padmanabhan V, Kasa-Vubu J, et al.: 1990. The role of biological activity of gonadotropins in puberty. In International Symposium on Developmental Endocrinology, Geneva, Switzerland.

Bogdanove EM, Nolin JM, Campbell GT: 1973. Qualitative and quantitative gonadpituitary feedback. Recent Prog Hormone Res 31:567-626.

Chappel SC, Ulloa-Aguirre A, Coutifaris C: 1983. Biosynthesis and secretion of follicle-stimulating hormone. Endocr Rev 4:179-211.

Dahl KD, Hsueh AJW: 1987. Use of the granulosa cell aromatase bioassay for measurement of bioactive follicle-stimulating hormone in urine and serum samples of diverse species. Steroids 50:375-392.
Dahl KD, Pavlou SN, Kovacs WJ, et al.: 1986. The changing ratio of serum bioactive to immunoreactive follicle-stimulating hormone in normal men following treatment with a potent gonadotropin releasing hormone antagonist. J Clin Endocrinol Metab 63:792-794.

Dahl KD, Czekala NM, Lim P, et al.: 1987. Monitoring the menstrual cycle of humans and lowland gorillas based on urinary profiles of bioactive follicle-stimulating hormone and steroid metabolites. J Clin Endocrinol Metab 64:486-493.

Dahl KD, Bicsak TA, Hsueh AJW: 1988a. Naturally occurring antihormones: secretion of FSH antagonists by women treated with a GnRH analog. Science 239:72-74.

Dahl KD, Jia X-C, Hsueh AJW: 1988b. Bioactive follicle-stimulating hormone levels in serum and urine of male and female rats from birth to prepubertal period. Biol Reprod 39:32-38.

Dufau ML, Veldhuis JD: 1987. Pathophysiological relationships between the biologi$\mathrm{cal}$ and immunological activities of luteinizing hormone. Clin Endocrinol Metab 1:153-176.

Fauser BCJM, Soto D, Czekala NM, et al.: 1989. Granulosa cell aromatase bioassay: changes of bioactive FSH levels in the female. I Steroid Biochem 33:721-726.

Fauser BCJM, Bogers JW, Hop WCJ, et al.: 1990. Bioactive and immunoreactive FSH in serum of normal and oligospermic men. Clin Endocrinol 32:433-442.

Hassing JM, Padmanabhan V, Kelch RP, et al.: 1990. Differential regulation of serum I-LH and B-FSH by testosterone in early pubertal boys. J Clin Endocrinol Metab 70:1082-1089.

Hsueh AJW, Bicsak TA, Jia X-C: 1989. Granulosa cells as hormone targets: the role of biologically active follicle-stimulating hormone in reproduction. Recent Prog Hormone Res 45:209-277.

Huhtaniemi IT, Dahl KD, Rannikko S, et al.: 1988. Serum bioactive and immunoreactive follicle-stimulating hormone in prostatic cancer patients during gonadotropinreleasing hormone agonist treatment and after orchidectomy. J Clin Endocrinol Metab 66:308-313.

Jia X-C, Hsueh AJW: 1985. Sensitive in vitro bioassay for the measurement of serum follicle-stimulating hormone. Neuroendocrinology 41:445-448.

Jia X-C, Kessel B, Yen SSC, et al.: 1986. Serum bioactive follicle-stimulating hormone during the human menstrual cycle and in hyper-and hypogonadotropic states: application of a sensitive granulosa cell aromatase bioassay. J Clin Endocrinol Metab 62:1243-1249.
Kessel B, Dahl KD, Kazer RR, et al.: 1988. The dependency of bioactive follicle-stimulating hormone secretion on gonadotropinreleasing hormone in hypogonadal and cycling women. J Clin Endocrinol Metab 66:361-366.

Montfort SL, Dahl KD, Czekala NM, et al.: 1989. Monitoring ovarian function and pregnancy in the giant panda (Ailuropoda melanoleuca) by evaluating urinary bioactive FSH and steroid metabolites. J Reprod Fertit 85:203-212.

Mortola JF, Sathanandan M, Pavlou S, et al.: 1989. Suppression of bioactive and immunoreactive follicle-stimulating hormone and luteinizing hormone levels by potent gonadotropin-releasing hormone antagonist: pharmacodynamic studies. Fertil Steril 51:957-963.

Padmanabhan V, Chappel SC, Beitins IZ: 1987. An improved in vitro bioassay for follicle-stimulating hormone (FSH): suitable for measurement of FSH in unextracted human serum. Endocrinology 121:10891098.

Padmanabhan V, Kelch RP, Sonstein J, et al.: 1988a. Bioactive follicle-stimulating hormone responses to intravenous gonadotropin-releasing hormone in boys with idiopathic hypogonadotropic hypogonadism. J Clin Endocrinol Metab 67:793800.

Padmanabhan V, Lang LL, Sonstein J, et al.: 1988b. Modulation of serum folliclestimulating hormone bioactivity and isoform distribution by estrogenic steroids in normal women and in gonadal dysgenesis. $\mathrm{J}$ Clin Endocrinol Metab 67:465-473.

Padmanabhan V, Sonstein J, Olton PL, et al.: 1989a. Serum bioactive follicle-stimulating hormone-like activity increases during pregnancy. J Clin Endocrinol Metab 69:968977.

Padmanabhan V, Ebling FJP, Sonstein J, et al.: 1989b. Bioactive follicle-stimulating hormone release in nutritionally growthretarded ovariectomized lambs: regulation by nutritional repletion. Endocrinology 125:2517-2526.

Pavlou S, Dahl KD, Wakefield G, et al.: 1988. Maintenance of the ratio of bioactive to immunoreactive follicle-stimulating hormone in normal men during chronic luteinizing hormone-releasing hormone agonist administration. J Clin Endocrinol Metab 66:1005-1009.

Reiter EO, Beitins IZ, Ostrea TR, et al.: 1982. Bioassayable luteinizing hormone during childhood and adolescence and in patients with delayed pubertal development. $\mathrm{J}$ Clin Endocrinol Metab 54:155-161.

Tenover JS, Dahl KD, Hsueh AJW, et al.: 1987. Serum bioactive and immunoreactive follicle- 
stimulating hormone levels and the response to clomiphene in healthy young and elderly men. J Clin Endocrinol Metab 64:1103-1108.

Urban RJ, Dahl KD, Padmanabhan V, et al.: 1991. Specific regulatory actions of dihydrotestosterone and estradiol on the dynamics of FSH secretion and clearance in man. J Androl 12:27-35.

Van Damme MP, Robertson DM, Marana R, et al.: 1979. A senstive and specific in vitro bioassay method for the measurement of FSH activity. Acta Endocrinol (Copenh) 91:224-237.

Walker LA, Cornell L, Dahl KD, et al.: 1988. Urinary concentrations of ovarian steroid hormone metabolites and bioactive folliclestimulating hormone in killer whales
(Orcinus orchus) during ovarian cycles and pregnancy. Biol Reprod 39:1013-1020.

Wang C: 1988. Bioassays of follicle-stimulating hormone. Endocr Rev 9:374-377.

Wang C, Dahl KD, Leung A, et al.: 1987. Serum bioactive follicle-stimulating hormone in men with idiopathic azoospermia and oligospermia. J Clin Endocrinol Metab 65:629-633.

Wang C, Zhong CQ, Leung A, et al.: 1990. Serum bioactive follicle-stimulating hormone levels in girls with precocious sexual development. J Clin Endocrinol Metab 70:615-619.

Wide L: 1986 . The regulation of metabolic clearance rate of human FSH in mice by variation of the molecular structure of the hormone. Acta Endocrinol (Copenh) 112:336344.

Wide L, Albertsson-Wikland K: 1990 . Change in electrophoretic mobility of human folliclestimulating hormone in serum after administration of gonadotropin-releasing hormone. $\mathrm{J}$ Clin Endocrinol Metab 70:271-276.

Wide L, Hobson BM: 1983. Qualitative difference in follicle-stimulating hormone activ ity in the pituitaries of young women compared to that of men and elderly women. J Clin Endocrinol Metab 56:371-375.

Wide L, Wide M: 1984. Higher plasma disappearance rate in the mouse for pituitary follicle-stimulating hormone of young women compared to that of men and elderly women. J Clin Endocrinol Metab 58:426-429.

\title{
Molecular Genetics of the Thyrotropin Receptor
}

\begin{abstract}
Gilbert Vassart, Marc Parmentier, Frédérick Libert, and Jacques Dumont
\end{abstract}

\begin{abstract}
Thyroid-stimulating hormone (TSH) receptor has been cloned by a strategy exploiting its belonging to the superfamily of G-protein-coupled receptors. Comparison with the luteinizing hormone and folliclestimulating hormone receptors shows that the glycoprotein hormone receptors constitute a subfamily of G-protein-coupled receptors with a large extracellular domain probably responsible for hormone binding. The recombinant human TSH receptor stably expressed in cell lines provides the tool to identify the targets of autoimmune reactions leading to Graves' disease and idiopathic myxedema. (Trends Endocrinol Metab 1991; 2:151-156)
\end{abstract}

Thyrotropin (thyroid-stimulating hormone, TSH), which is the main regulator of the thyroid gland [reviewed by Dumont et al. (1989a)], is responsible for the rapid control of thyroid function (thyroid hormone secretion), maintains

Gilbert Vassart is at the Institute of Interdisciplinary Research, and at the Faculty of Medicine and Service of Medical Genetics, Free University, Brussels, Belgium. Marc Parmentier, Frédérick Libert, and Jacques Dumont are at the Institute of Interdisciplinary Research, Brussels, Belgium. the differentiated state of the gland (for example, expression of the thyroglobulin and thyroperoxidase genes, as well as iodide transport), and is the major growth factor for thyrocytes (Dumont et al. 1989b). In humans and dogs, at least, all of these TSH effects can be mimicked by the forskolin-induced rise of intracellular cyclic (c) AMP. Although this does not exclude a role for other regulatory cascades (in particular, the phospholipase C-phosphoinositides-calcium system) or for other growth factors (such as epidermal growth factor, EGF), it is well accepted that modulation by TSH of the adenylyl-cyclase-protein-kinase-A system represents the principal pathway of thyroid regulation (Dumont et al. 1989a).

The concept of a TSH receptor in the plasma membrane and the experimental evidence for its existence provided a rationale for the pathophysiology of Graves' disease and for some forms of idiopathic myxedema. Autoantibodies directed against the TSH receptor can either activate it, causing goiter and hyperthyroidism, or interfere with TSH binding and action, leading to involution of the gland and hypothyroidism (ReesSmith et al. 1988).

Its pivotal role in the understanding of thyroid physiology and pathology, and in the development of diagnostic assays for stimulating or blocking autoantibodies, prompted numerous studies aiming at the characterization, isolation, and finally cloning of the TSH receptor. Whereas a coherent picture of the receptor emerged rather early from affinitylabeling studies, mainly from the group of Rees-Smith (1988), the cloning attempts have long encountered failures, false leads, and frustration. This longlasting fallow period has now yielded to an abundance of information. Following our initial report (Parmentier et al. 1989), several groups have now cloned the TSH receptor almost simultaneously (Nagayama et al. 1989; Misrahi et al. 1990; Lindsay et al. 1990; Akamizu et al. 1990), exploiting breakthroughs in the related fields of G-protein-coupled receptors or LH-CG receptor cloning (Loosfelt et al. 1989; McFarland et al. 1989). 